\title{
Speaker Abstracts From ASENT 2004 Annual Meeting March 11-13, 2004
}

\section{Translational Neuroscience: From Molecules to Humans}

Floyd E. Bloom, M.D.

Elsewhere (see Bloom, Science 300:1680-1685, 2003) I have commented upon the extraordinarily precarious state of the american health system, and asserted that the impending collapse of this system presents a gigantic obstacle to the application of the discoveries flowing from biomedical research. Unless steps are taken soon to undertake a comprehensive restoration of the health system, the profound advances in biomedical research so rapidly accruing today may never be effectively transformed into meaningful advances in health care for society both in the United States and globally. Supporters of postgenomic medicine have touted entry into a time when physicians will be able to recognize an individual's vulnerabilities to inheritable, disease-causing factors early enough in their lifespan to be able to help those individuals prevent the onset of their diseases. Even though most human heritable neurological and psychiatric diseases are not the result of single dominant or recessive genetic mutations, the studies of strongly inheritable diseases including alcoholism, drug dependencies, depression, multiple sclerosis, and deafness have provided solid clues to help understand the sporadic and complex, multigenic diseases. Current trends in preclinical neuroscience reveal that we are now taking some early steps in the transition from symptomand disease-driven medicine to a predictive, preemptive, preventive postgenomic medicine. These steps will be illustrated by recent results with transgenic mice as models for amyotrophic lateral sclerosis, Alzheimer's disease, and depression, and the development of methods to detect and quantitate early cellular neuropathologies and refine them into biomarkers suitable for early intervention assessments. Nevertheless, the very skills and time that will be necessary for the wise clinicians of the future to invest in the study of individual patterns of disease progression and their responses to therapeutic and preventive interventions will not be welcomed by profit-driven, highthroughput care systems. If medications of the future are to be molecularly tailored to individual needs, and acceptable to the regulatory drug approval process that demands risk-free efficacy, the scientific community must now unite to plan for such a realizable future.

\section{Paradoxes in Equivalence Trials-It Is Time to Readjust Your Intuition}

\section{Richard Chappell, Ph.D.}

Equivalence trials are clinical experiments designed to assess whether a new treatment is similar enough to a standard agent that the two may be considered equivalent by some measure. Interest in them is escalating because they are now frequently performed to justify the use of new regimens. In addition, the International Conference on Harmonization ( $\mathrm{ICH})$ guideline E-10, "Choice of Control Group in Clinical Trials," has accelerated interest since its recent publication.

ICH E-10 states: "There are major differences in practice and attitudes toward the need for placebo controlled trials...and the acceptability of active control equivalence trials as evidence of efficacy and safety." There are a number of design considerations that apply to equivalence trials in an intrinsically different manner than they do to superiority trials (if, in fact, they are relevant to the latter at all). Issues of properly treating historical data, the use of the intent-to-treat principle and whether it is still conservative, and the proper formulation of a null hypothesis all deserve reexamination in this context. This talk shows that some of the accepted knowledge that has been developed with respect to traditional randomized clinical trials does apply to equivalence studies, some of it is inapplicable, and much of it is applicable only with caution and after thorough examination.

\section{Industry Perspective on the Use of Placebos and Active Controls in Clinical Trials}

Mark Corrigan, M.D.

The randomized, placebo-controlled clinical trial has become the gold standard of scientific evidence for assessing the efficacy and safety of new therapeutics. From a historical perspective, the evolution of the placebo controlled trial is a relatively recent concept. Despite the scientific advantages of this design, the Helsinki declaration of 2000 has brought controversy to whether placebo-controlled trials are ethical at all. Interpretations of the declaration have varied by geographic region, limiting clinical trials to certain countries and disease states. Placebo-controlled trials allow for more rapid assessment of the benefits and risks of new medicines, an essential feature for the pharmaceutical industry, faced with spiraling research and development expenditures. An additional hurdle of the establishment of comparative efficacy and safety for new therapeutics continues to evolve, as active control studies become necessary to obtain pricing and reimbursement beyond the traditional phase IV market expansion studies.

\section{Functions of the Proteasome: From Cell Biology to Cancer Therapy \\ Alfred L. Goldberg, M.D.}

Most proteins in mammalian cells are degraded by the ubiquitin-proteasome pathway, where protein substrates are linked to ubiquitin molecules by one of the many ubiquitin ligases (E3s) of the cell. This modification marks the protein for rapid degradation by the $26 \mathrm{~S}$ proteasome. This large complex uses ATP to unfold the proteins and to inject them into its 20S core particle, where they are digested to small peptides. This system selectively destroys abnormally folded proteins, as they unfortunately accumulate in various neurodegenerative diseases, and many regulatory proteins, important in the control of gene expression and growth.

Peptides released by proteasomes range from 2 to 24 residues in length. Although most are rapidly digested to amino acids, some are transported through the endoplasmic reticulum to the cell surface, where they are presented to the immune system on major histocompatibility complex class I molecules. This process enables circulating cytotoxic $\mathrm{T}$ cells to screen for and eliminate virally infected cells and cancers.

With denervation, in many systemic diseases (e.g., cancer) 
and fasting, muscles atrophy due to a general activation of the ubiquitin-proteasome pathway in muscles. The atrophying muscles show a common pattern of changes in expression of specific genes (which we term "atrogenes"). The two proteins induced most dramatically are muscle-specific ubiquitin ligases, atrogin-1 and MuRF-1, which trigger the atrophy process and the accelerated proteolysis.

Much has been learned about the functions of this system by the use of inhibitors of the proteasome that enter cells and inhibit intracellular proteolysis. Blocking proteasome function eventually induces apoptosis, especially in cancer cells. One such inhibitor (Velcade PS341) has recently been approved by the Food and Drug Administration for treatment of multiple myeloma, but it is now in many phase II trials against diverse cancers.

\section{Pathogenic Mechanisms as Therapeutic Targets in Parkinson's Disease}

J. Timothy Greenamyre, M.D., Ph.D.

Parkinson's disease (PD) may be caused, in rare instances, by either single genetic mutations or by single toxic exposures. Most cases, however, appear to result from a combination of an individual's overall genetic susceptibility and a lifetime of environmental exposures. As our understanding of the pathogenesis of PD improves, it is becoming apparent that a limited set of pathogenic mechanisms is operative in both the genetic and the sporadic forms of the disease.1) Mitochondrial impairment, especially at the level of complex I, has been implicated from both genetic and toxicological studies (e.g., rotenone and MPTP). Preliminary work suggests the complex I defect may be amenable to targeted gene transfer therapy. 2) Additionally, because mitochondrial impairment and other factors produce oxidative damage to proteins and DNA, there is reason to believe that lipid-soluble, brain-penetrant antioxidants will be protective. 3) $\alpha$-synuclein, a protein of uncertain function, causes PD both when it is mutated and when the wild-type protein is simply overexpressed. Thus, genetic or pharmacological downregulation of $\alpha$-synuclein may provide a neuroprotective strategy, especially in families in which genetic alterations of $\alpha$-synuclein are causative. 4) Genetic studies have implicated proteasomal dysfunction in PD pathogenesis, because both parkin and UCHL1 are components of the ubiquitin-proteasome system (UPS). UPS impairment can be blocked with antioxidants in animal models, raising the possibility that such compounds might be neuroprotective. In summary, relatively few pathogenic mechanisms appear to be central to the neurodegenerative process in PD. Targeting these mechanisms individually, or in combination, may provide a way to slow or halt the progression of PD.

\section{A Participant's Perspective on Placebos}

$$
\text { Jeffrey Martin, Esq. }
$$

Although the current regulatory environment encourages investigators in clinical trials to advise prospective participants that the purpose of the trial is to advance science rather than to help them individually, it is clear that participating in clinical trials by those approached to participate in them does involve in many cases an individually oriented cost-benefit analysis. Jeffrey C. Martin will discuss this from a patient's perspective and discuss how the regulatory environment and financial arrangements that influence participation in clinical trials should be examined in light of that utilitarian perspective.

\section{$\boldsymbol{\beta}$-Amyloid Peptide as an Immunotherapeutic Treatment for Alzheimer's Disease}

Dale Schenk, Ph.D.

Alzheimer's disease is the leading cause of dementia. The $\beta$-amyloid peptide, the core constituent of neuritic plaques found in the brain tissue of patients suffering from the disease, has been identified as a possible causative agent in the pathophysiology of the disease. A variety of therapeutic strategies are aimed at either reducing the production of this peptide (i.e., $\beta$ or $\gamma$ secretase inhibitors) or improving its clearance from the brain. Recently, immunization with $\beta$-amyloid peptide was proven remarkably effective in APP transgenic mouse models of the disease in both preventing the occurrence of plaques and reducing existing pathology in older animals. This immunotherapeutic effect of $\mathrm{A} \beta$ appears to be mediated by antibodies against the peptide. These findings have fostered efforts to test this strategy clinically for the possible treatment of Alzheimer's disease.

\section{Doubling Your Pleasure: The Placebo Effect in Neurological Illness}

A. Jon Stoessl, M.D.

As in other conditions affecting the nervous system, the placebo effect in Parkinson's disease is prominent, and may in some situations be sustained over several months. Using positron emission tomography with the dopamine receptor ligand $\left[{ }^{11} \mathrm{C}\right]$ raclopride (RAC), we have demonstrated that the placebo effect in Parkinson's is mediated by the release of endogenous dopamine. Several issues of interest arise from our findings: 1) the magnitude of the effect is considerable, and comparable to the response that is seen following administration of amphetamine to healthy control subjects; 2) patients who perceived subjective benefit following administration of placebo released a greater amount of dopamine into the dorsal (motor) striatum than those who did not perceive improvement in their parkinsonian symptoms; 3) all patients demonstrated a comparable degree of dopamine release in the ventral striatum, regardless of the degree of subjective benefit. Together, these findings suggest that the expectation of benefit is associated with the release of dopamine in areas of the brain involved in reward signaling, whereas the perception of benefit is mediated by dopamine release in the area of the brain most affected by Parkinson's. The critical role of expectation suggests that dopamine release in the ventral striatum could potentially contribute to mediation of the placebo effect in conditions other than Parkinson's, and also explains the failure of some studies to demonstrate a robust placebo effect. Clinical trials should take into account the fact that certain study designs are more likely than others to maximize the placebo effect (and could potentially thereby mask a true effect of the treatment under investigation). Furthermore, our finding that the effect of placebo is additive to that of active treatment suggests that the placebo effect can be harnessed to maximize therapeutic responses in a real-life setting.

\section{Regulatory Perspective on the Use of Placebo and Active Controls}

\section{Robert J. Temple, M.D.}

The choice of control group (placebo control $v s$ active control in a noninferiority study) poses inferential and ethical problems. The ethical issue is when it is acceptable to deny 
patients known available effective therapy by randomizing some of them to a placebo. The inferential question is when an active control noninferiority trial can actually demonstrate effectiveness. In general, when available treatment prevents serious harm (death, irreversible morbidity) a placebo cannot be used, but when there is no such harm, well informed patients can be asked to participate in a placebo-controlled trial, even if they may experience discomfort as a result (ICH E-10, "Choice of Control Group in Clinical Trials").

The problem with an equivalence or noninferiority study is that there is a critical assumption that may be incorrect, namely that the study could have detected a difference between two treatments that differed in effectiveness, e.g., an active drug and a placebo. It may seem intuitively obvious that almost all well designed and conducted studies would be able to do so, but that is not true. In many symptomatic conditions (depression, anxiety, allergic rhinitis, symptoms of heart failure), effective drugs often do not show an effect greater than placebo.

In practice, a noninferiority study seeks to show that the difference between the control and test drug (favoring the control) is not larger than some margin (the noninferiority margin).
The margin cannot be larger than the effect of the control drug in the study (if the difference between control and test drug favoring control were greater than the whole effect of the control, then the test drug would have no effect at all). Put another way, the $95 \%$ confidence interval for the term C-T must be less than the margin $\mathrm{M}$, ruling out a difference greater than M. But the effect of the control is not measured in the new study; it must be estimated from past placebo-controlled studies, which is not possible if the controls do not almost always show superiority to placebo. If an active control study could not have detected a difference between active and inactive drugs [because the active drug might not have had an effect of defined size (M) in that study], the study is said to lack "assay sensitivity." In that case, ruling out difference of $\mathrm{M}$ would not demonstrate an effect of the test drug. To be informative, a noninferiority study must have assay sensitivity and have a defined, supportable noninferiority margin reflecting the assured effect of the control in that study. Where drugs don't regularly distinguish themselves from placebo (depression, anxiety, dementia, seasonal allergies, etc.), noninferiority studies cannot provide evidence of an effect. 